\title{
Inhibition of hedgehog signaling depresses self-renewal of pancreatic cancer stem cells and reverses chemoresistance
}

\author{
FENG-TING HUANG $^{1 *}$, YONG-XUN ZHUAN-SUN ${ }^{2 *}$, YAN-YAN ZHUANG $^{1}$, \\ SHU-LI WEI $^{3}$, JIAN TANG ${ }^{1}$, WEN-BO CHEN ${ }^{1}$ and SHI-NENG ZHANG ${ }^{1}$ \\ Departments of ${ }^{1}$ Gastroenterology, and ${ }^{2}$ Respirology, Sun Yat-Sen Memorial Hospital, Sun Yat-Sen University, \\ Guangzhou, Guangdong 510120; ${ }^{3}$ Department of Oncology, The Affiliated Chen-Xinghai Hospital \\ of Guangdong Medical College, Xiaolan, Zhongshan, Guangdong 528415, P.R. China
}

Received May 8, 2012; Accepted July 20, 2012

DOI: $10.3892 /$ ijo.2012.1597

\begin{abstract}
Pancreatic cancer stem cells play a crucial role in tumorigenesis and chemoresistance. The Hedgehog signaling pathway is a key regulator in pancreatic tumorigenesis and drug resistance. To identify pancreatic cancer stem cells, tumorspheres derived from the PANC-1 pancreatic cancer cell line were cultured under a floating-culture system. PANC-1 tumorspheres possessed properties of self-renewal, differentiation, higher tumorigenesis and chemoresistance. It was observed that Hedgehog pathway is active in PANC-1 tumorspheres as shown by expression of hedgehog components Smo, Gil 1 and Gli 2, detected by quantitative RT-PCR and western blotting. After cyclopamine-mediated blockade of hedgehog, a decrease in proliferation of PANC-1 tumorspheres and $G_{0} / G_{1}$ transition were observed, as well as a decreased expression of Bmi-1 in PANC-1 tumorspheres. Cyclopamine reversed chemoresistance to gemcitabine, resulting in decreased expression of ABCG2 in PANC-1 tumorspheres. Taken together, our data indicate that PANC-1 tumorspheres have 'stemness' potential, and hedgehog signaling pathway plays an important role in the regulation of self-renewal and reversal of chemoresistance in cancer stem cells in pancreatic adenocarcinoma.
\end{abstract}

\section{Introduction}

Pancreatic cancer (PC) is one of the most malignant tumors. It is the fourth leading cause of cancer death in the United States (1) and has an increasing trend of mortality rate in China (2).

Correspondence to: Dr Shi-Neng Zhang, Department of Gastroenterology, Sun Yat-Sen Memorial Hospital, Sun Yat-Sen University, No. 107 Yanjiang West Road, Guangzhou, Guangdong 510120, P.R. China

E-mail: shinengz@hotmail.com

${ }^{*}$ Contributed equally

Key words: pancreatic cancer, tumorsphere, cancer stem cell, hedgehog signaling pathway, ABCG2, Bmi-1
Due to the lack of obvious symptoms or specific manifestation in the early stage, the disease is often diagnosed too late to have available therapeutic intervention options.

Cancer stem cells (CSCs) are a small subpopulation of multipotent cells exhibiting self-renewal capacity, multilineage differentiation and high carcinogenesis $(3,4)$, and have been identified in several types of human cancers including pancreatic cancer (5-8). Tumorsphere under suspension culture is one of the important techniques to isolate cancer stem cell. Dysregulation of CSC self-renewal may lead to expansion of the stem-like cells, which might result in early-stage carcinogenesis $(9,10)$. Chemoresistance of CSCs is one of the most critical reasons leading to failure of chemotherapy, which might be followed by tumor recurrence.

Hedgehog (Hh) family members are key regulators of carcinogenesis $(4,11)$. Hh signal transduction is initiated by binding Hh ligand, including Shh, Dhh and Ihh, to its receptor Patched (PTCH). In the absence of $\mathrm{Hh}$ protein, $\mathrm{PTCH}$ represses signal transduction by inhibiting Smoothened (Smo). When Hh ligand binds to PTCH receptor, inhibitory effects of PTCH on Smo diminish, Hh pathway is activated and the ultimate step is mediated by the zinc finger transcription factors-Gli family which turns on genes regulating cell cycle, and determinating cell-fate. Hh pathway is active in many tumors, such as medulloblastoma (12), glioma (13), gastric cancer (14) and pancreatic cancer (15). Increasing evidence supports that hedgehog pathway has a role in the maintenance and progression of pancreatic cancer (16-18). It also has a crucial role in reverse chemoresistance of some CSCs, such as CD34+ leukaemic cells (19) and glioblastoma CSCs (20), but the mechanism is still unclear.

The breast cancer resistance protein (BCRP/ABCG2), a member of the G-subfamiliy of the ATP-binding cassette (ABC)-transporter superfamily, is implied to be associated with multidrug resistance in some cancer stem cells, such as side population in lung cancer (21), and prostate tumorshpere (22). In some cancers, there is relationship between $\mathrm{Hh}$ pathway and ABCG2. In diffuse large B-cell lymphoma (23), ABCG2 is the target of Hh pathway.

In the present study, we isolated pancreatic tumorspheres under floating-culture system, and identified the stemness potential of self-renewal, differentiation, high carcinogenesis and chemoresistance. We elucidated the important role of Hedgehog 
pathway in regulation of self-renewal and chemoresistance of pancreatic CSCs.

\section{Materials and methods}

Cell lines and animals. The human pancreatic adenocarcinoma cell line PANC-1 (Chinese Academy of Sciences, Shanghai, China) was cultured in RPMI-1640 (Gibco, Grand Island, NY, USA) supplemented with $10 \%$ fetal bovine serum (FBS, HyClone, Logan, UT, USA), $100 \mathrm{U} / \mathrm{ml}$ penicillin G, and $100 \mu \mathrm{g} /$ $\mathrm{ml}$ streptomycin. In all experiments, cells were maintained at $37^{\circ} \mathrm{C}$ in a humidified $5 \% \mathrm{CO}_{2}$ air atmosphere. Male non-obese diabetic/severe combined immunodeficiency (NOD/SCID) mice (6-8 weeks) were purchased from Chinese Academy of Sciences (Beijing, China). Animal care and experimental protocols were performed in accordance with procedures and guidelines established by Chinese Academy of Sciences Experimental Animal Care Commission.

Expansion of PANC-1 tumorspheres. Viable, floating single cells were collected from the supernatant of PANC-1 cells by centrifugation at $1,000 \mathrm{rpm}$ for $5 \mathrm{~min}$ and plated at 1,000 cells/ $\mathrm{ml}$ in serum-free DMEM-F12 (Gibco) supplemented with $20 \mathrm{ng} / \mathrm{ml}$ recombinant human epidermal growth factor (rhEGF, Invitrogen, NY, USA), $0.4 \%$ bovine serum albumin (BSA, Sigma, St. Louis, MO, USA), $5 \mu \mathrm{g} / \mathrm{ml}$ insulin, 1:50 B27 supplement (Gibco, Invitrogen), $100 \mathrm{U} / \mathrm{ml}$ penicillin, and $100 \mathrm{mg} / \mathrm{ml}$ streptomycin. Cells grown in this condition as non-adherent spheres were enzymatically dissociated after 12 days by incubation in a trypsin-EDTA solution and then cultured to generate tumorspheres of the next generation. Differentiation was induced by culturing tumorsphere-derived cells in RPMI-1640 supplemented with $10 \%$ fetal bovine serum without rhEGF.

Sphere formation assay. PANC-1 tumorspheres and its parental cell line PANC-1 were dissociated, and 1,000 cells per well were plated in 6-well culture dishes in $5 \mathrm{ml}$ DMEM-F12 medium with rhEGF. The number of spheres for each well was evaluated after 15 days of culture.

Immunofluorescence analysis. To detect the differential potential of PANC-1 tumorspheres, the spheres were cultured in DMEMF12 supplemented with $10 \%$ FBS. Observation of morphology was performed by microscope. The expression of CK18, a differentiation marker, was detected by immunofluorescence on 1st, 5th and 10th day. The cells were washed with PBS and fixed in $4 \%$ paraform for $15 \mathrm{~min}$ on ice. After two more phosphatebuffered solution (PBS) washes, the cells were covered with $0.5 \%$ Triton-100 for 15 min on ice, then washed with PBS and incubated with $5 \%$ non-fat milk for $1 \mathrm{~h}$ at room temperature to block non-specific binding of IgG. The cells were incubated with primary antibody anti-human CK18 (Cell Signaling Technology, Boston, MA, USA) for $2 \mathrm{~h}$ at room temperature, then washed with PBS and incubated with fluorochrome-conjugated secondary antibody at room temperature for $30 \mathrm{~min}$ in a dark chamber. The cells were washed with PBS and covered with DAPI to stain the nuclei. Random photographs were taken at x200 magnification.

Tumorsphere xenografts. To explore the tumorigenic capacity, PANC-1 tumorsphere cells in concentrations ranging from 200 to $2 \times 10^{4}$ were injected into the subcutaneous space of the pad of NOD/SCID mice. Tumor growth was monitored every 2 days after the second week of inoculation. When the xenograft tumors had reached the desired size, mice were sacrificed and a portion of the tumor tissue was collected, fixed in $4 \%$ paraform, and embedded in paraffin for hematoxylin and eosin (H\&E) staining to assess tumor pathology. Tumors of three animals were harvested per experiment.

Quantitative real-time $R T-P C R(q R T-P C R)$. To analyse the hedgehog pathway components, qRT-PCR was performed to examine the expression level of Smo, Gli 1 and Gli 2 mRNA in PANC-1 tumorspheres and PANC-1. Briefly, total RNA was extracted from the cells using TRIzol (Invitrogen, CA, USA) according to the manufacturer's instructions. For qRT-PCR, $1 \mu \mathrm{l}$ of gene primers with SYBR Green in $20 \mu \mathrm{l}$ of reaction volume was applied. $18 \mathrm{~S}$ ribosomal RNA was used as an endogenous control. Primers were designed as: Smo, forward, 5'-cat ccc tga ctg tga gat ca-3'; reverse, 5'-cac cat ctt ggt gac atg ct-3'. Gli 1, forward, 5'-cca tac atg tgt gag cac ga-3'; reverse, 5'-ggc aca gtc agt ctg ctt t-3'. Gli 2, forward, CAA CGC CTA CTC TCC CAG AC; reverse, GAG CCT TGA TGT ACT GTA CCA C. QRT-PCR was performed with SYBR Green PCR System (Toyobo). Amplification data were analyzed with Applied Biosystems Prism Sequence Detection Software version 2.1 (Applied Biosystems).

Cell proliferation assay by WST-8 method. PANC-1 tumorspheres were harvested and dissociated into single cell suspension, 3x10 4 PANC-1 sphere cells were seeded in 96-well plate per well. PANC-1 was also dissociated into single cell suspension, $3 \times 10^{3}$ cells were seeded in 96 -well plate per well. The cells were treated in different concentration of cyclopamine (Sigma-Aldrich, St. Louis, MO) $(0,0.5,1,2,5$ and $10 \mu \mathrm{mol} / 1$, respectively) in triplicate for 24,48 and $72 \mathrm{~h}$, respectively. WST-8 reagent $(10 \mu \mathrm{l}$ per well) from Cell Counting kit- 8 (Dojindo, Kumamoto, Japan) was added, incubated for $4 \mathrm{~h}$, and absorbance was determined with a multi-well spectrophotometer (BioTek, VT, USA $)$ at 450 and $630 \mathrm{~nm}$. Inhibition rate $=(1-$ absorbance of treated cells/control cells) x100\%.

Western blot analysis. The concentration of total protein extracted from PANC-1 and PANC-1 tumorsphere cells with and without cyclopamine was determined with a BCA Protein Assay kit (Pierce, USA). Equal amounts of protein were separated by $10 \%$ SDS-PAGE and electrophoretically transferred to PVDF membranes (Millipore, Bedford, MA, USA) using a mini trans-blot. Rat anti-human CK18 (Cell Signaling Technology), Bmi-1 (Abcam, MA, USA), ABCG2 (Abcam), Smo (Abcam), rabbit anti-human Gli 1 (Santa Cruz Biotechnology, Santa Cruz, CA, USA) and Gli 2 (Santa Cruz Biotechnology) were used to detect the expression of homologous proteins. $\beta$-actin (Santa Cruz Biotechnology) was used as an internal control. Electrochemiluminescence was performed with a Chemilmager 5500 imaging system (San Leandro, CA, USA), according to the manufacturer's instructions.

Flow cytometry analysis. To detect the influence of tumorspheres on cell cycle after treatment of cyclopamine, flow cytometry analysis was performed. Briefly, tumorsphere cells and PANC-1 


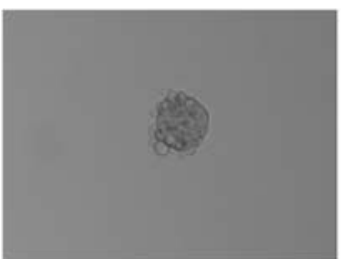

Day 5

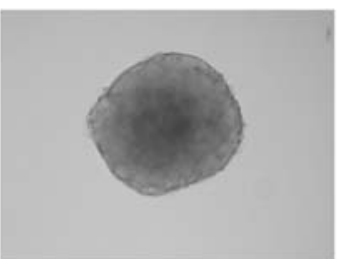

Day 10

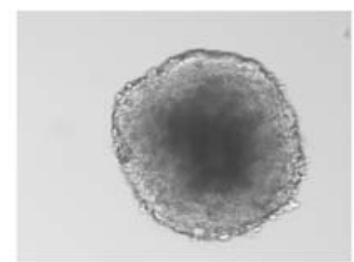

Day 15

Figure 1. Morphology of the 1st PANC-1 tumorsphere. Tumorspheres were incubated under a floating-cultured system, with growth factor and without bovine serum. Tumorspheres began to form on day 5. Tumorspheres were propagated on the 10th and 15th day. 1st spheres could be enzymatically dissociated into single cells, which in turn generated the 2 nd spheres. The procedure could be repeated and the PANC-1 spheres have been passed over 20 generations in vitro in the present study.

were treated with $10 \mu \mathrm{mol} / \mathrm{l}$ cyclopamine for $72 \mathrm{~h}$. Then the cells were trypsinized and fixed with ice-cold $70 \%$ ethanol for $18 \mathrm{~h}$ at $4^{\circ} \mathrm{C}$. The fixed cells were stained with $50 \mathrm{mg} / \mathrm{ml}$ propidium iodide (BD Pharmingen, San Diego, CA) and $50 \mathrm{mg} / \mathrm{ml}$ RNase and then analysed using a flow cytometer (BD Pharmingen). PANC-1 tumorspheres and PANC-1 without cyclopamine incubation were performed as control.

CD133 is implied as a stem cell marker in many tumors, to detect phenotypic difference of CD133 in PANC-1 and PANC-1 spheres, cells were harvested with trypsin-EDTA to produce a single cell suspension. The cells were pelleted by centrifugation, washed twice with PBS and incubated with FITC conjugated CD133 (Ancell, Bayport, MN, USA) for $30 \mathrm{~min}$ in the dark. The cells were washed twice with PBS after incubation and analyzed using a flow cytometer.

Statistical analysis. Data are presented as mean \pm standard deviation (SD), using the SPSS software, version 11.0. The means were then compared using a one-way ANOVA with LSD among groups or Student's t-test between groups. $\mathrm{P}<0.05$ was considered statistically significant.

\section{Results}

PANC-1 tumorspheres cultured under floating-culture system possess self-renewal potential. One of the probable method to identify the CSCs is serum-free floating-culture system. It was sucessfully used to culture neural stem cell and other cancer stem cells, such as breast CSCs and brain CSCs. We applied this method to isolate PANC-1 tumorspheres from human pancreatic cancer cell line PANC-1. The adherent PANC-1 was trypsinized into single-cell suspension, and was seeded in serum-free media supplemented with growth factors at clonal density (1,000 cells/ $\mathrm{ml})$. Five days later, PANC-1 tumorspheres began to form in culture and they propagated after 10-15 days (Fig. 1). The 1st spheres could be enzymatically dissociated into single cells, which in turn generated the 2 nd spheres. This procedure could be repeated and the PANC-1 tumorspheres have been passaged over 20 times in vitro.

We subsequently examined whether PANC-1 tumorsphere cells possess self-renewal capacity. Sphere formation assay was performed to calculate the number of stem cell spheres and measure the self-renewal capacity of each sphere generation. Each generation of tumorsphere cells $\left(10^{3}\right)$ were plated in 6-well dish, and cultured for 15 days in triplicate. The 1st PANC-1
Table I. Tumor formation initiated by PANC-1 tumorsphere cells in NOD/SCID mice.

\begin{tabular}{lccccc}
\hline & \multicolumn{5}{c}{ Dilutions (cells/ml) } \\
\cline { 2 - 6 } $\begin{array}{l}\text { Cell type of } \\
\text { inoculation }\end{array}$ & $2 \times 10^{2}$ & $2 \times 10^{3}$ & $2 \times 10^{4}$ & $2 \times 10^{5}$ & $2 \times 10^{6}$ \\
\hline $\begin{array}{l}\text { Tumorsphere } \\
\text { cells }\end{array}$ & $0 / 3$ & $1 / 3$ & $3 / 3$ & - & - \\
PANC-1 & - & - & $0 / 3$ & $2 / 3$ & $3 / 3$ \\
\hline
\end{tabular}

tumorsphere cells form 5.5 \pm 1.3 spheres; The 4th PANC-1 tumorsphere cells form $4.5 \pm 1.3$ spheres $(\mathrm{P}>0.05)$. The capacity of serial-passage in vitro and the number of tumorspheres that remained equivalent show the self-renewal potential of PANC-1 tumorspheres.

PANC-1 tumorspheres have differentiation potential. Differentiation potential is one of the capacites of CSCs. CK18 is a mature marker associated to luminal/ductal epithelial cells, and acts as a differentiation marker in this study. Both immunofluorescence analysis and western blot analyses were performed to detect the expression of CK18. To induce differentiation, floating PANC-1 tumorspheres were cultured in the medium without growth factors and with $10 \%$ fetal bovine serum. Under differentiating conditions, floating PANC-1 tumorspheres began to adhere and acquired epithelium-like morphology. Expression of CK18 was increased after induction of differentiation (Fig. 2).

PANC-1 tumorspheres have higher tumorigenic potential. High tumorigenic potential is one of the characteristics of CSCs. To detect the tumorigenicity of PANC-1 tumorspheres, a concentration ranging from 200 to $2 \times 10^{4}$ tumorsphere cells were injected into the subcutaneous space of the pad of NOD/SCID mice. After 11 weeks, $2 \times 10^{3}$ PANC-1 tumorsphere cells initiated a tumor in 1 of 3 NOD/SCID mice (Fig. 3). Whereas, $\geq 10^{5}$ PANC-1 cells initiated a tumor in NOD/SCID mice (Table I). The data imply that PANC-1 tumorsphere cells are more tumorigenic than its parental cell line. Collectively, floating PANC-1 tumorspheres have potential of self-renewal, differentiation and high tumorigenesis, which are the characteritics of CSCs. 


\section{A \\ Microscope}

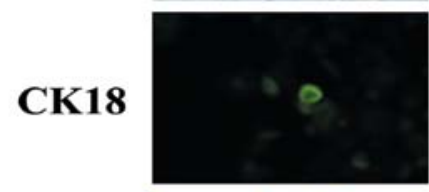

DAPI
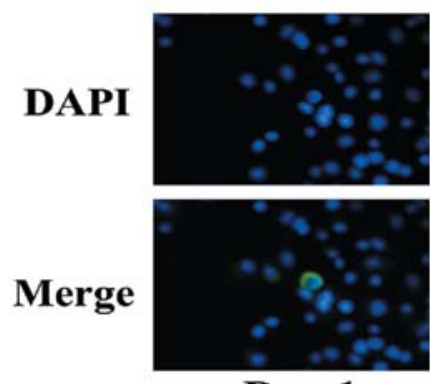

Day 1
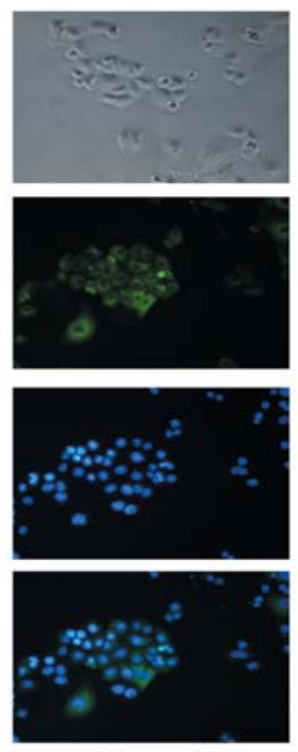

Day 5
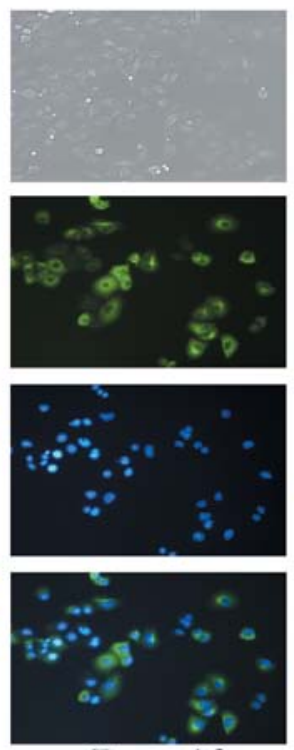

Day 10

\section{B}

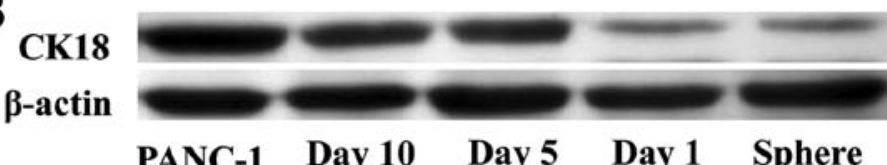

Figure 2. Morphology of PANC-1 tumorspheres under differentiation condition and immunofluorescence and western blot analysis of expression of CK18. (A) Morphology of PANC-1 tumorspheres under differentiation condition and immunofluorescence analysis of expression of CK18. Tumorspheres were cultured without growth factor and with $10 \%$ fetal bovine serum. Floating PANC-1 tumorspheres began to adhere and acquired epithelium-like morphology gradually on the 5th and 10th day. (B) Western blot analysis of CK18. CK18 is a mature marker associated to luminal/ductal epithelial cells, and acts as a differentiation marker. with time, increased expression of CK18 was detected. This suggested that PANC-1 tumorspheres had differentiation potential.
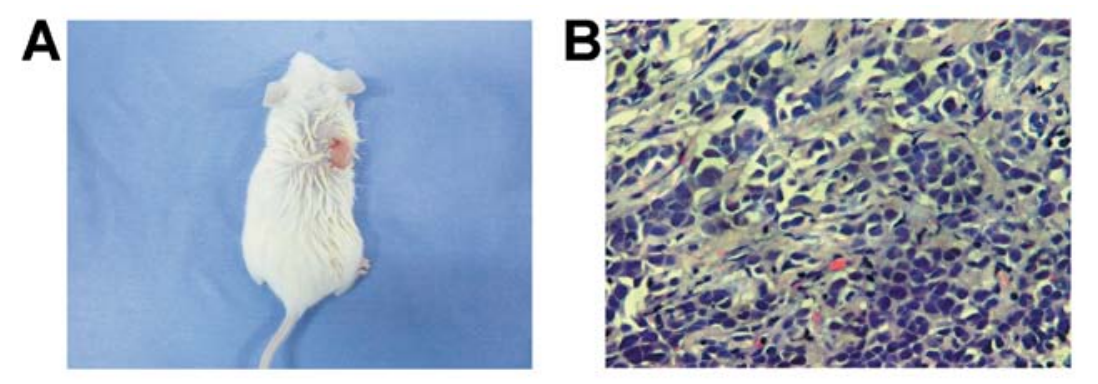

Figure 3. Xenograft of PANC-1 tumorspheres in NOD/SCID. (A) Tumor formation in NOD/SCID mice when $2 \times 10^{3}$ PANC-1 spheres cells were injected. (B) Hematoxylin-eosin staining of a section through PANC-1 sphere cell xenograft. x200 magnification.
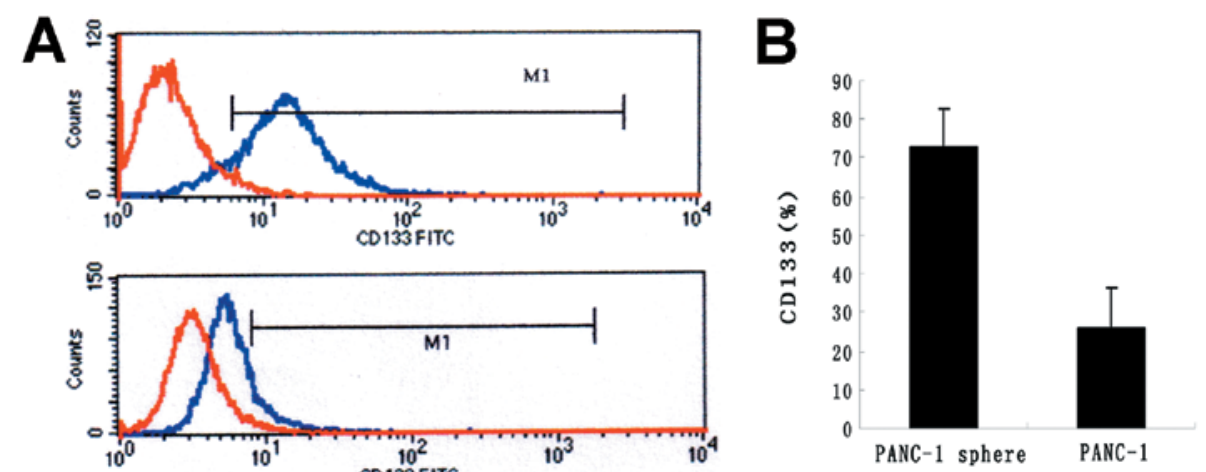

Figure 4. Expression of CD133 of PANC-1 sphere and PANC-1. (A) Determination of CD133 expression by flow cytometry. (B) Statistical analysis of expression of CD133 shown in (A). The CD133 expression in PANC-1 spheres was significantly higher than that in PANC-1. The fraction of CD133 in PANC-1 sphere is $(72.73 \pm 4.38) \%$, while the fraction of CD133 in PANC-1 is $(26.16 \pm 2.13) \%(\mathrm{P}<0.05)$. 


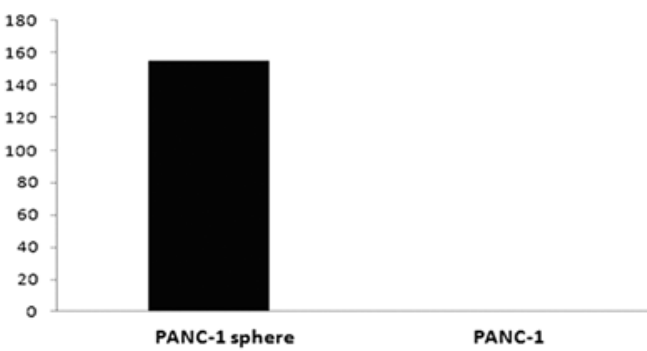

B
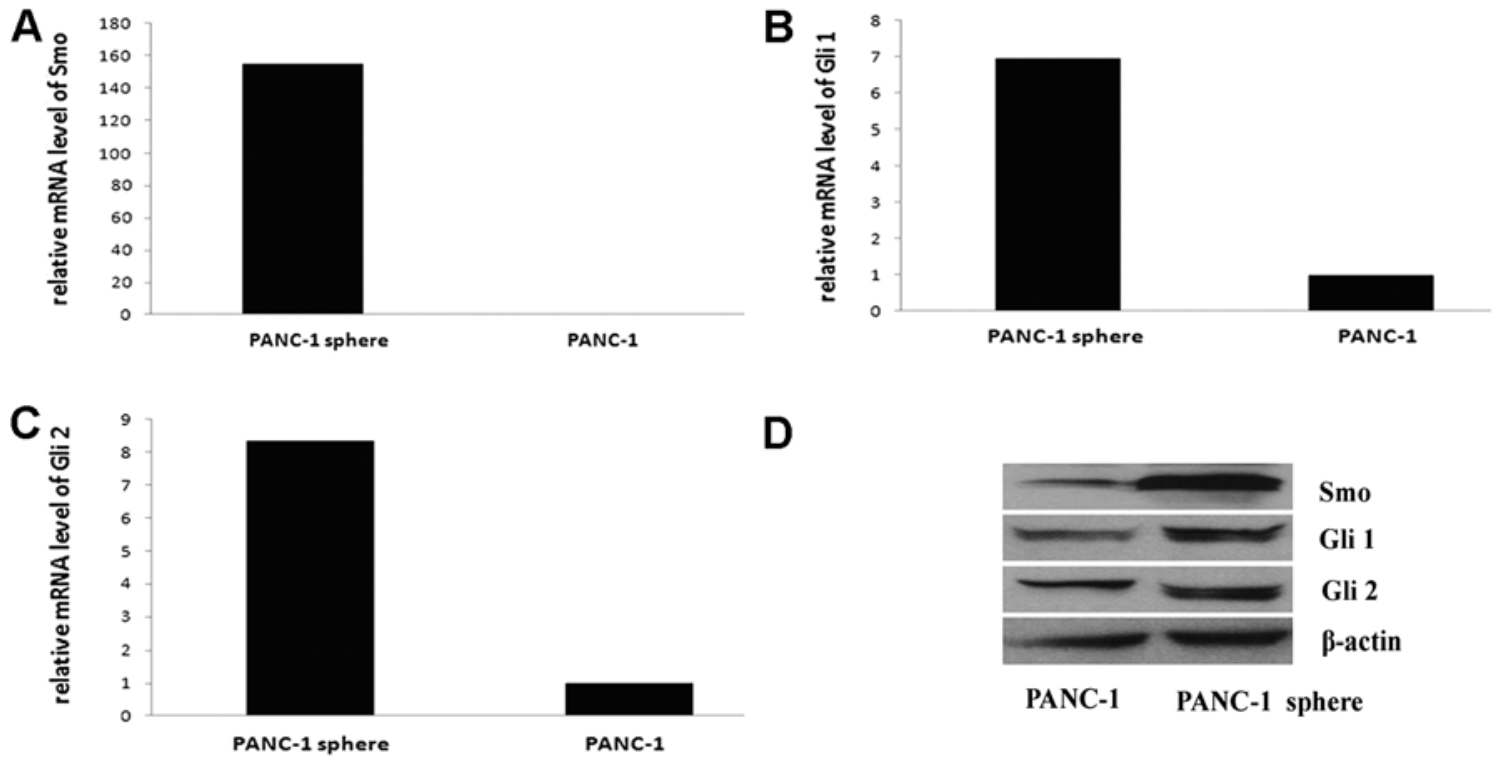

D

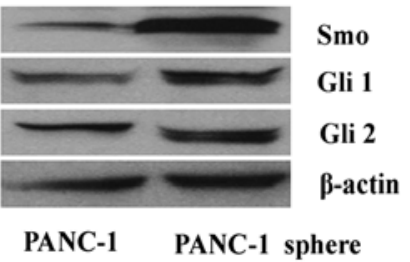

Figure 5. Expression of Smo, Gli 1 and Gli 2 mRNA and protein in the PANC-1 tumorspheres and PANC-1 by qRT-PCR and western blot analysis. (A) qRT-PCR analysis of relative expression levels of Smo. (B) qRT-PCR analysis of relative expression levels of Gli 1. (C) qRT-PCR analysis of relative expression levels of Gli 2. (D) Western blot analysis of expression levels of Smo, Gli 1 and Gli 2.

CD133 is highly expressed in PANC-1 tumorspheres. CD133 is well-known as stem cell marker in many tumors. To detect the phenotypic difference between PANC-1 tumorspheres and PANC-1, FACS was carried out. The $\mathrm{CD} 133^{+}$subfraction was dramatically increased in PANC-1 tumorspheres compared with PANC-1 (72.73 \pm 4.38 vs $26.16 \pm 2.13 \%)(\mathrm{P}<0.05)$ (Fig. 4).

The Hh pathway is active in PANC-1 tumorspheres. Hh pathway is active in some CSCs, such as glioma CSCs and breast CSCs. Also, Hh pathway is active in pancreatic ductal adenocarcinoma. To evaluate the possibility that Hh signaling is active in pancreatic CSCs, we examined the expression of Smo, Gli 1 and Gli 2 mRNA and protein in PANC-1 tumorspheres and PANC-1 cells by qRT-PCR and western blotting. Hh pathway is active in both PANC-1 tumorspheres and PANC-1 cells, Smo, Gli 1and Gli 2 mRNA are high expressed, especially in PANC-1 tumorspheres, the expression of Smo mRNA is 154.76-fold as that of PANC-1 cells, the expression of Gli 1 mRNA is 6.94-fold that of PANC-1 cells and Gli 2 is 8.36-fold. The results of western blot analysis are consistent with the mRNA expression (Fig. 5).

Cyclopamine-mediated blockade of Hh pathway inhibits proliferation of PANC-1 CSCs via Bmi-1. To detect the effects on inhibition of Hh pathway, we used cyclopamine, a special inhibitor of Smo, to block the Hh pathway. PANC-1 tumorsphere cells were treated in different concentration of cyclopamine $(0,0.5,1,2,5$ and $10 \mu \mathrm{mol} / 1$, respectively) in triplicate for different times (24, 48 and $72 \mathrm{~h}$ ). Cyclopaminemediated Hh pathway blockade inhibited the overall growth rate of the culture in a dose-and time-dependent fashion. After treatment with $10 \mu \mathrm{mol} / 1$ cyclopamine for $72 \mathrm{~h}$, the inhibition rate of PANC-1 tumorsphere cells was $(37.85 \pm 13.69) \%$ $(\mathrm{P}<0.05)$. Furthermore, to investigate whether cell cycle of PANC-1 tumorsphere cells change after cyclopamine treatment, flow cytometry was performed to detect the cell cycle of tumorspheres with and without cyclopamine. After incuba- tion with $10 \mu \mathrm{mol} / \mathrm{l}$ cyclopamine for $72 \mathrm{~h}$, percentage of $\mathrm{G}_{0} /$ $\mathrm{G}_{1}$ of PANC-1 tumorspheres was decreased $(36.53 \pm 6.03) \%$, compared to $(67.41 \pm 6.35) \%$ before incubation $(\mathrm{P}<0.05)$ (Fig. $6 \mathrm{~A}$ and $\mathrm{B})$. We hypothesized that Bmi-1 may function as a downstream target of the Hh pathway. To test the hyposis, we examine the expression of Bmi-1 protein of PANC-1 tumorspheres with and without cyclopamine incubation by western blotting. After incubation with $10 \mu \mathrm{mol} / 1$ cyclopamine for 72 $\mathrm{h}$, expression of Bmi-1 protein was reduced (Fig. 6C).

Cyclopamine reverses chemoresistance of PANC-1 tumorspheres via $A B C G 2$. Drug sensitivity assay was carried out to detect the chemotherapy resistance of PANC-1 tumorspheres and its parental cell line PANC-1. PANC-1 tumorspheres are more resistant to gemcitabine and 5-FU than PANC-1. $\mathrm{IC}_{50}$ of gemcitabine in PANC-1 tumorspheres is $500.75 \pm 10.51 \mu \mathrm{g} / \mathrm{ml}$, while PANC-1 is $11.43 \pm 2.10 \mu \mathrm{g} / \mathrm{ml}(\mathrm{P}<0.05) . \mathrm{IC}_{50}$ of $5-\mathrm{FU}$ in PANC-1 tumorspheres and PANC-1 is $224.37 \pm 5.71 \mu \mathrm{g} / \mathrm{ml}$ vs $2.19 \pm 0.32 \mu \mathrm{g} / \mathrm{ml}(\mathrm{P}<0.05)$. Expression of ABCG 2 is detected by western blotting. As shown, expression of ABCG2 in PANC-1 tumorspheres is much higher than that of PANC-1 (Fig. 7). Moreover, we investigated whether inhibition of Hh pathway could reverse chemoresistance of PANC-1 tumorspheres, and we used cyclopamine to block the Hh pathway. PANC-1 tumorsphere cells preincubated with $10 \mu \mathrm{mol} / 1$ cyclopamine for $72 \mathrm{~h}$ were treated with different concentration of gemcitabine $(0,0.5$, $5,25,50,100,500$ and $1000 \mu \mathrm{g} / \mathrm{ml}$ ) for $72 \mathrm{~h}$. $\mathrm{IC}_{50}$ of gemcitabine in PANC-1 tumorspheres with and without cyclopamine is $124.55 \pm 12.37 \mu \mathrm{g} / \mathrm{ml}$ vs $500.75 \pm 10.51 \mu \mathrm{g} / \mathrm{ml}(\mathrm{P}<0.05)$. We hypothesized the expression of ABCG2 in PANC-1 tumorsphere cells changed after cyclopamine treatment. To consolidate this notion, western blot analysis was performed to detect expression of ABCG2 protein of tumorspheres with and withdrawl cyclopamine. PANC-1 tumorsphere after incubation with cyclopamine displayed significantly reduced levels of ABCG2 compared to tumorspheres without cyclopamine incubation $(\mathrm{P}<0.05)$ (Fig. 8). 

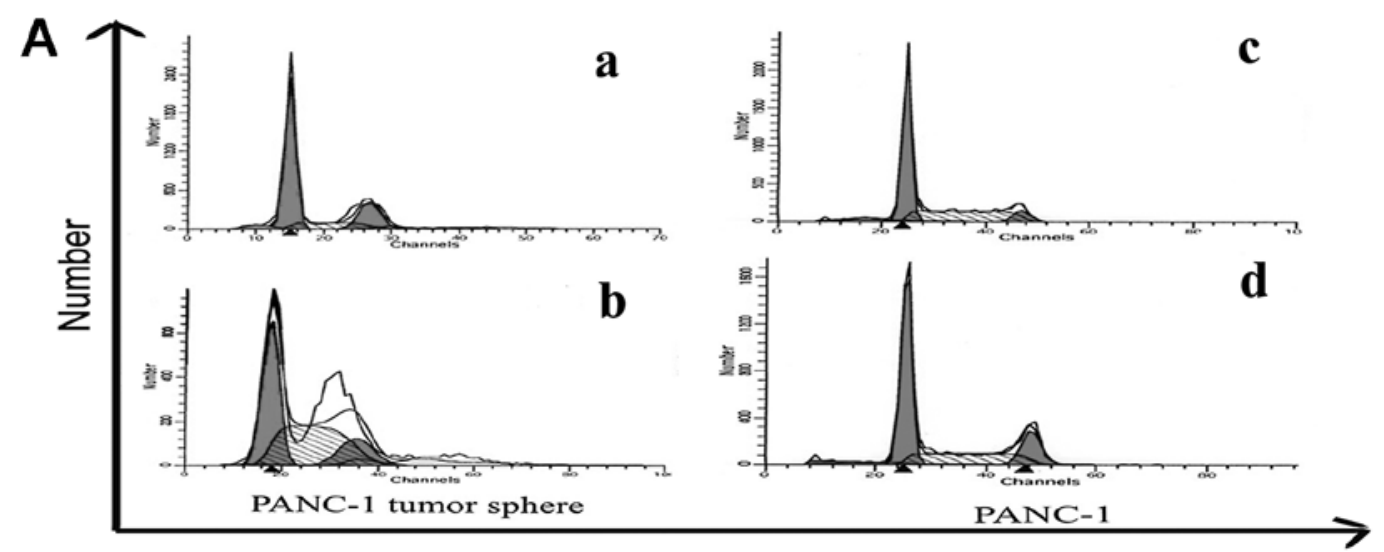

B
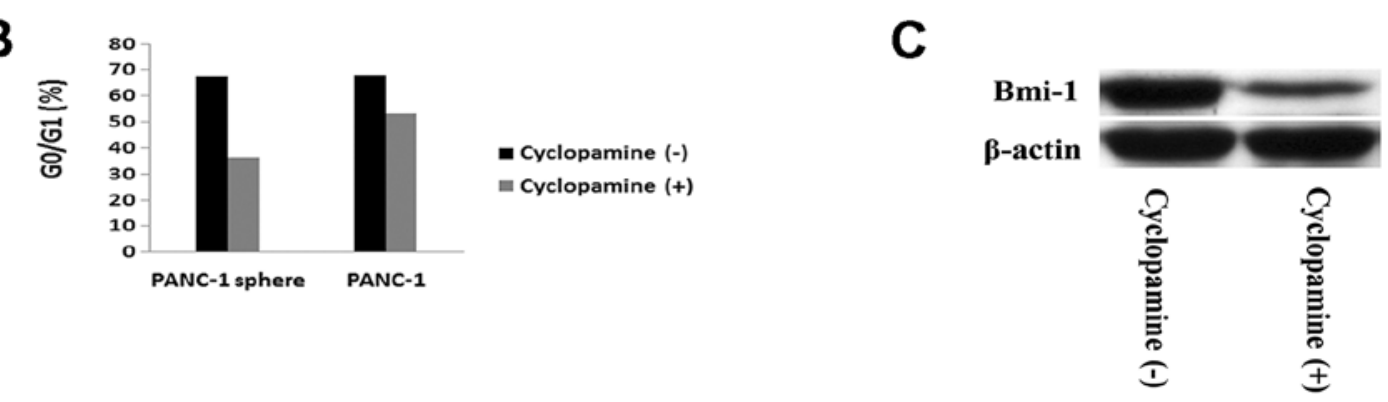

Figure 6. (A) Cell cycle of the PANC-1 tumorsphere and PANC-1 with and without cyclopamine. Cell cycle of PANC-1 tumorsphere without cyclopamine (a) and with cyclopamine (b), PANC-1 without cyclopamine (c) and with cyclopamine (d). (B) Statistical analysis of fraction of $\mathrm{G}_{0} / \mathrm{G}_{1}$ in PANC-1 tumorsphere and PANC-1. (C) Western blot analysis of Bmi-1 in PANC-1 tumorsphere with and without cyclopamine. After incubation with $10 \mu \mathrm{mol} / 1 \mathrm{cyclopamine}$ for $72 \mathrm{~h}$, percentage of $\mathrm{G}_{0} / \mathrm{G}_{1}$ of PANC-1 tumorspheres declined to $36.53 \pm 6.03 \%$, compated to $67.41 \pm 6.35 \%$ before incubation (P<0.05). Fraction of $\mathrm{G}_{0} / \mathrm{G}_{1}$ of $\mathrm{PANC}-1$ was decreased to $53.13 \pm 1.10 \%$, compared to $67.64 \pm 6.88 \%$ before incubation $(\mathrm{P}<0.05)$. Furthermore, after incubation with $10 \mu \mathrm{mol} / 1 \mathrm{cyclopamine}$ for $72 \mathrm{~h}$, PANC-1 tumorsphere displayed a significantly reduced level of Bmi-1 compared to tumorsphere without cyclopamine.
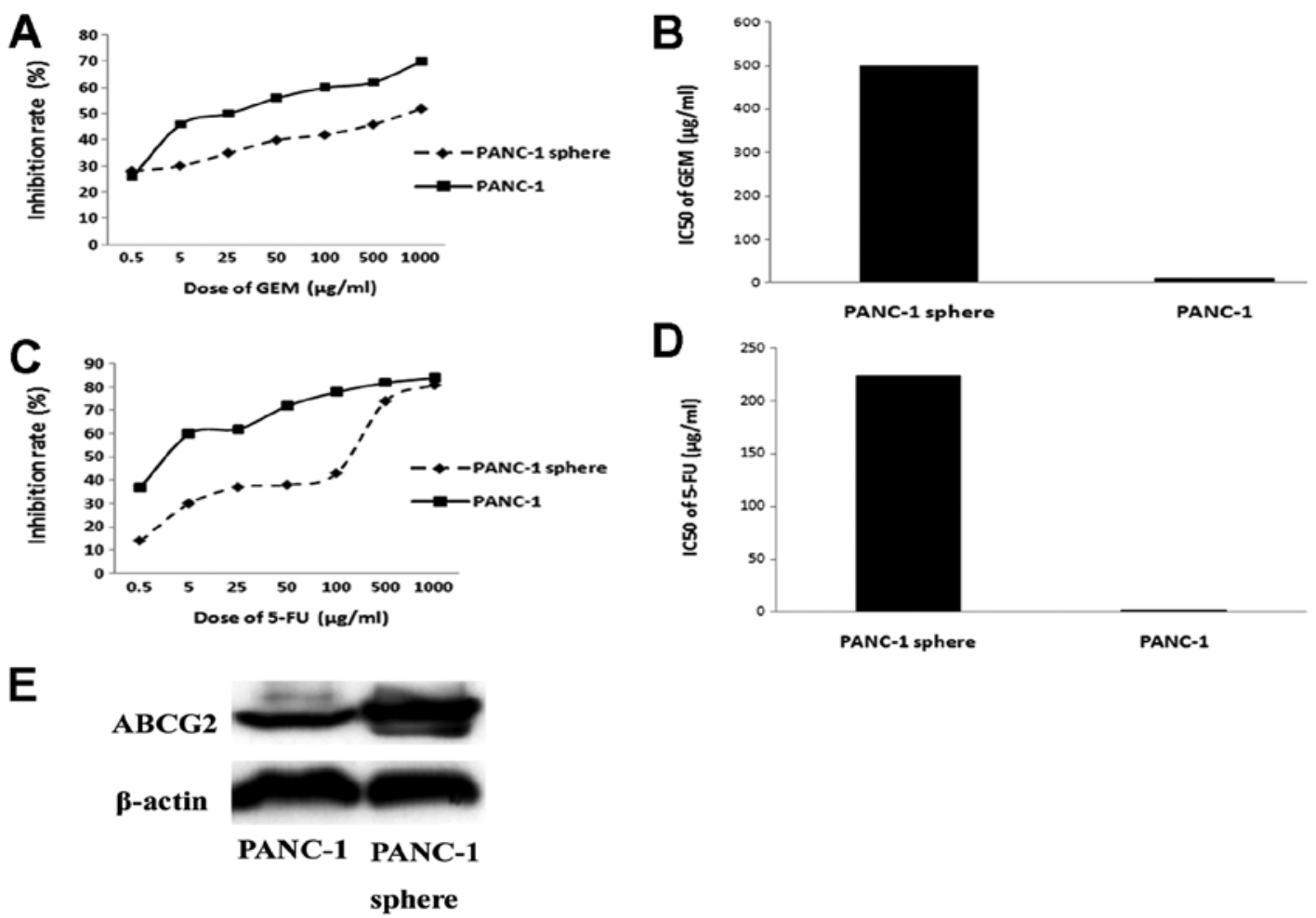

Figure 7. Drug sensitivity assay of PANC-1 sphere and PANC-1. (A) Inhibition curve of PANC-1 sphere and PANC-1 in various dose of gemcitabine (GEM). (B) Statistical analysis of IC $_{50}$ of GEM in PANC-1 sphere and PANC-1. (C) Inhibition curve of PANC-1 sphere and PANC-1 in various dose of 5-FU. (D) Statistical analysis of $\mathrm{IC}_{50}$ of 5-FU in PANC-1 sphere and PANC-1. (E) Western blot analysis of ABCG2 in PANC-1 sphere and PANC-1. PANC-1 spheres are more resistant to GEM and 5-FU than PANC-1. IC $_{50}$ of GEM in PANC-1 spheres is 500.75 $\pm 10.51 \mu \mathrm{g} / \mathrm{ml}$, while PANC-1 is $11.43 \pm 2.10 \mu \mathrm{g} / \mathrm{ml}(\mathrm{P}<0.05)$. $\mathrm{IC}_{50}$ of 5-FU in PANC-1 spheres and PANC-1 is $224.37 \pm 5.71 \mu \mathrm{g} / \mathrm{ml}$ vs $2.19 \pm 0.32 \mu \mathrm{g} / \mathrm{ml}(\mathrm{P}<0.05)$. PANC-1 spheres demonstrated a significantly enhanced level of ABCG2 compared to PANC-1. 


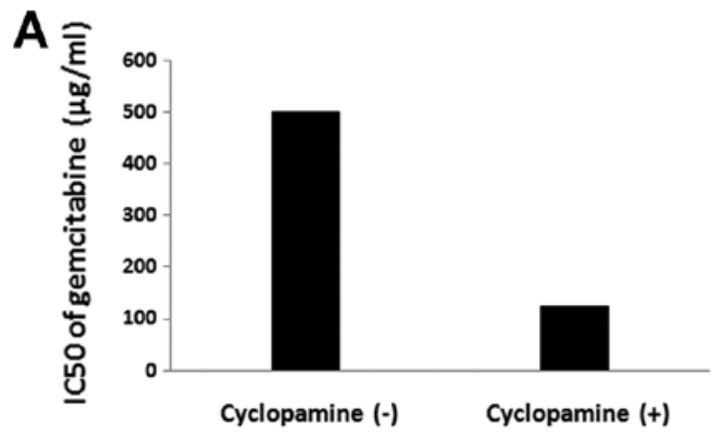

B

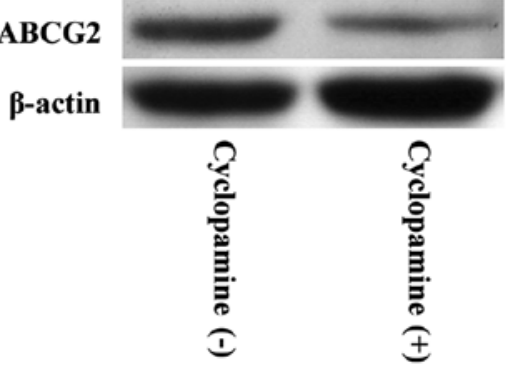

Figure 8. Drug sensitivity assay of PANC-1 sphere with and without cyclopamine. (A) Statistical analysis of $\mathrm{IC}_{50}$ of GEM in the PANC-1 sphere with and without cyclopamine. (B) Western blot analysis of ABCG2 in PANC-1 sphere with and without cyclopamine. $\mathrm{IC}_{50}$ of GEM in PANC-1 spheres with and without cyclopamine is $124.55 \pm 12.37 \mathrm{vs} 500.75 \pm 10.51 \mu \mathrm{g} / \mathrm{ml}(\mathrm{P}<0.05)$. Expression of ABCG2 in PANC-1 spheres declined significantly after incubation with cyclopamine. Blockade of the Hh pathway in PANC-1 sphere can reverse chemoresistance via ABCG2.

\section{Discussion}

Accumulating evidence has demonstrated that a tumor is in essence heterogeneous and contains cancer stem cells which possess the potential of self-renewal, multilineage differentiation, high proliferation, and chemoresistance. More and more studies support existance of tumor-initiating cells in pancreatic adenocarcinoma $(7,8)$. Serum-free floating culture system has been used to identify CSCs in several cancer types such as brain cancer, breast cancer and colon cancer (24-26). The cells isolated using this system were proved to have the stem cell features and represent the small population in tumors which was the reason of tumor formation, metastasis and resistance to chemotherapy. The advantage of this strategy to enrich CSCs is its independence of specific cell surface markers. In our study, we isolated PANC-1 tumorspheres under floating-culture system, and it could be serially propagated in vitro over 20 passages. Sphere formation assay showed that the number of spheres kept equivalent when being propagated in vitro. Under differentiating conditions, PANC-1 tumorspheres were adherent and differentiated. They acquired epithelial morphology and expressed mature marker CK18 associated to luminal/ductal cells. More CK18 was expressed when the time of differentiation was extended. Furthermore, as few as $2 \times 10^{3}$ PANC-1 sphere cells initiated a tumor in NOD/SCID mice, a 100-fold enhanced tumorigenic potential compared to its parental cell line, which is in accordance with Li et al (7). Taken together, PANC-1 tumorspheres displayed potential of self-renewal, differentiation and high tumorigenicity, which are the characteristics of cancer stem cells.

Hedgehog signal pathway is active in pancreatic cancer (27), also it is active in many CSCs, such as prostate CSCs (28), and glioblastoma CSCs (20). Hedgehog has been associated with the self-renewal process of CSCs (29). B lymphoma Mo-MLV insertion region 1 (Bmi-1), a transcriptional repressor belonging to the polycomb group (PCG) of transcription factors, has been reported to play a key role in regulating self-renewal of leukemic stem cell (30) and breast CSCs (23). It is reported that the Hh pathway regulates selfrenewal of human medulloblastoma brain tumor-initiating cells via Bmi-1 (31). In our study, inhibition of Hh pathway depressed self-renewal of the PANC-1 tumorsphere. To illustrate whether $\mathrm{Hh}$ regulates $\mathrm{PANC}-1$ tumorsphere via Bmi-1, we examined the expression of Bmi-1 with and without cyclopamine-mediated blockade of $\mathrm{Hh}$. Expression of Bmi-1 was reduced after blockade of the Hh pathway. Taken together, these data suggested that Hedgehog pathway and Bmi-1 might be involved in pancreatic tumorigenesis.

It is reported that CSCs are naturally chemoresistant (32). ABCG2 with drug capability are preferentially expressed in CSCs. Also, the chemoresistant phenotype of CSCs can be mediated by ABCG2 protein. Hh pathway was illustrated to have a relationship with chemoresistance of pancreatic cancer stem cells (33), and ABCG2 is reported to be the direct transcriptional target of $\mathrm{Hh}$ pathway in drug tolerance in diffuse large B-cell lymphoma (34). In our study, we found that PANC-1 tumorspheres were more resistant to gemcitabine and 5-FU compared to PANC-1. Expression of ABCG2 was significantly elevated in PANC-1 tumorspheres. Blockade of Hh pathway by cyclopamine reversed chemoresistance of gemcitabine in PANC-1 tumorspheres. Moreover, expression of ABCG2 was decreased after cyclopamine incubation. Hence, it was implied in our study that inhibition of $\mathrm{Hh}$ pathway reversed chemoresistance in PANC-1 tumorspheres via ABCG2.

In conclusion, our study illustrated that tumorspheres derived from pancreatic cancer cell line PANC-1 possessed self-renewal, chemoresistance and other stemness properties. Hh pathway was active in PANC-1 tumorspheres. More importantly, our data suggested that inhibition of Hh pathway depressed self-renewal of pancreatic CSCs via Bmi-1, which might be involved in pancreatic tumorigenesis. Our study also demonstrated the crucial role of Hh pathway of pancreatic CSCs in mediating chemotherapy resistance associated with ABCG2.

\section{Acknowledgements}

We would like to thank Dr Jing Wei for assistance with flow cytometry. This research was supported in part by a grant from the Natural Science Foundation of Guangdong Province (no. 815100890100013, 04009381) and Medical Scientific Research Foundation of Guangdong Province (no. B2009066). 


\section{References}

1. Greenlee RT, Murray T, Bolden S and Wingo PA: Cancer statistics. CA Cancer J Clin 50: 7-33, 2000.

2. Wang L, Yang GH, Lu XH, Huang ZJ and Li H: Pancreatic cancer mortality in China (1991-2000). World J Gastroenterol 9: 1819-1823, 2003

3. Reya T, Morrison SJ, Clarke MF and Weissman IL: Stem cells, cancer, and cancer stem cells. Nature 414: 105-111, 2001.

4. Beachy PA, Karhadkar SS and Berman DM: Tissue repair and stem cell renewal in carcinogenesis. Nature 432: 324-331, 2004.

5. Al-Hajj M, Wicha MS, Benito-Hernandez A, Morrison SJ and Clarke MF: Prospective identification of tumorigenic breast cancer cells. Proc Natl Acad Sci USA 100: 3983-3988, 2003.

6. Singh SK, Hawkins C, Clarke ID, et al: Identification of human brain tumour initiating cells. Nature 432: 281-282, 2004.

7. Li CW, Heidt DG, Dalerba P, et al: Identification of pancreatic cancer stem cells. Cancer Res 67: 1030-1037, 2007.

8. Hermann PC, Huber SL, Herrler T, et al: Distinct populations of cancer stem cells determine tumor growth and metastatic activity in human pancreatic cancer. Cell Stem Cell 1: 241-242, 2007.

9. Dontu G, Al-Hajj M, Abdallah WM, Clarke MF and Wicha MS: Stem cells in normal breast development and breast cancer. Cel Prolif 36: 59-72, 2003.

10. Wicha MS, Liu S and Dontu G: Cancer stem cells: an old idea - a paradigm shift. Cancer Res 66: 1883-1890, 2006.

11. Berman DM, Karhadkar SS, Maitra A, et al: Widespread requirement for hedgehog ligand stimulation in growth of digestive tract tumors. Nature 425: 846-851, 2003.

12. Berman DM,Karhadkar SS,Hallahan AR, et al: Medulloblastoma growth inhibition by hedgehog pathway blockade. Science 297 : $1559-1561,2002$.

13. Clement V, Sanchez P, de Tribolet N, Radovanovic I and Ruiz i Altaba A: HEDGEHOG-GLI1 signaling regulates human glioma growth, cancer stem cell self-renewal, and tumorigenicity. Curr Biol 17: 165-172, 2007.

14. Han ME, Lee YS, Baek SY, Kim BS, Kim JB and Oh SO: Hedgehog signaling regulates the survival of gastric cancer cells by regulating the expression of Bcl-2. Int J Mol Sci 10: 3033-3043, 2009.

15. Thayer SP, di Magliano MP, Heiser PW, et al: Hedgehog is an early and late mediator of pancreatic cancer tumorigenesis Nature 425: 851-856, 2003.

16. Jones S, Zhang X, Parsons DW, et al: Core signaling pathways in human pancreatic cancers revealed by global genomic analyses. Science 321: 1801-1806, 2008.

17. Feldmann G, Habbe N, Dhara S, et al: Hedgehog inhibition prolongs survival in a genetically engineered mouse model of pancreatic cancer. Gut 57: 1420-1430, 2008.

18. Jimeno A, Feldmann G, Suárez-Gauthier A, et al: A direct pancreatic cancer xenograft model as a platform for cancer stem cell therapeutic development. Mol Cancer Ther 8: 310-314, 2009 .
19. Liu S, Dontu G, Ilia D. Mantle ID, et al: Hedgehog signaling and Bmi-1 regulate self-renewal of normal and malignant human mammary stem cells. Cancer Res 66: 6063-6071, 2006.

20. Bar EE, Chaudhry A, Lin A, et al: Cyclopamine-mediated hedgehog pathway inhibition depletes stem-like cancer cells in glioblastoma. Stem Cells 25: 2524-2533, 2007.

21. Shi Y, Fu X, Hua Y, Han Y, Lu Y and Wang J: The side population in human lung cancer cell line NCI-H460 is enriched in stem-like cancer cells. PLoS One 7: e33358, 2012.

22. Zhang L, Jiao M, Li L, et al: Tumorspheres derived from prostate cancer cells possess chemoresistant and cancer stem cell properties. J Cancer Res Clin Oncol 138: 675-686, 2012.

23. Park IK, Qian D, Kiel M, et al: Bmi-1 is required for maintenance of adult self-renewing haematopoietic stem cells. Nature 423: 302-305, 2003.

24. Inagaki A, Soeda A, Oka N, et al: Long-term maintenance of brain tumor stem cell properties under at non-adherent and adherent culture conditions. Biochem Biophys Res Commun 361: 586-592, 2007.

25. Ponti D, Costa A, Zaffaroni N, et al: Isolation and in vitro propagation of tumorigenic breast cancer cells with stem/progenitor cell properties. Cancer Res 65: 5506-5511, 2005.

26. Ricci-Vitiani L, Lombardi DG and Pilozzi E: Identification and expansion of human colon-cancer-initiating cells. Nature 445: 111-115, 2007.

27. Inaguma S, Kasai $\mathrm{K}$ and Ikeda H: GLI1 facilitates the migration and invasion of pancreatic cancer cells through MUC5ACmediated attenuation of E-cadherin. Oncogene 30: 714-723, 2011.

28. Chang HH, Chen BY, Wu CY, et al: Hedgehog overexpression leads to the formation of prostate cancer stem cells with metastatic property irrespective of androgen receptor expression in the mouse model. J Biomed Sci 18: 6, 2011.

29. Takebe N, Harris PJ, Warren RQ and Ivy SP: Targeting cancer stem cells by inhibiting Wnt, Notch, and Hedgehog pathways. Nat Rev Clin Oncol 8: 97-106, 2011.

30. Dimri GP, Martinez JL, Jacobs JJ, et al: The Bmi-1 oncogene induces telomerase activity and immortalizes human mammary epithelial cells. Cancer Res 62: 4736-4745, 2002.

31. Wang X, Venugopal C, Manoranjan B, et al: Sonic hedgehog regulates Bmil in human medulloblastoma brain tumor-initiating cells. Oncogene 31: 187-199, 2012.

32. Rich JN and Bao S: Chemotherapy and cancer stem cells. Cell Stem Cell 1: 353-355, 2007

33. Yao J, An Y, Wie JS, et al: Cyclopamine reverts acquired chemoresistance and down-regulates cancer stem cell markers in pancreatic cancer cell lines. Swiss Med Wkly 141: w13208, 2011.

34. Singh RR, Kunkalla K, Qu C, Schlette E, Neelapu SS, Samaniego F and Vega F: ABCG2 is a direct transcriptional target of hedgehog signaling and involved in stroma-induced drug tolerance in diffuse large B-cell lymphoma. Oncogene 30 : 4874-4886, 2011. 